OPEN ACCESS

Edited by:

Christine Ladd-Acosta,

Johns Hopkins University,

United States

Reviewed by: Jeffrey Mark Craig,

Royal Children's Hospital, Australia

*Correspondence:

Janine M. LaSalle

jmlasalle@ucdavis.edumailto:

jmlasalle@ucdavis.edu

Specialty section:

This article was submitted to

Neurogenomics,

a section of the journal

Frontiers in Genetics

Received: 08 December 2021

Accepted: 28 January 2022

Published: 15 February 2022

Citation:

Mouat JS and LaSalle JM (2022) The

Promise of DNA Methylation in

Understanding Multigenerational

Factors in Autism Spectrum Disorders.

Front. Genet. 13:831221

doi: 10.3389/fgene.2022.831221

\section{The Promise of DNA Methylation in Understanding Multigenerational Factors in Autism Spectrum Disorders}

\author{
Julia S. Mouat ${ }^{1,2,3,4}$ and Janine M. LaSalle ${ }^{1,2,3,4 *}$ \\ ${ }^{1}$ LaSalle Laboratory, Department of Medical Microbiology and Immunology, University of California, Davis, Davis, CA \\ United States, ${ }^{2}$ Perinatal Origins of Disparities Center, University of California, Davis, Davis, CA, United States, ${ }^{3}$ MIND Institute, \\ School of Medicine, University of California, Davis, Davis, CA, United States, ${ }^{4}$ Genome Center, University of California, Davis, \\ Davis, CA, United States
}

Autism spectrum disorder (ASD) is a group of neurodevelopmental disorders characterized by impairments in social reciprocity and communication, restrictive interests, and repetitive behaviors. Most cases of ASD arise from a confluence of genetic susceptibility and environmental risk factors, whose interactions can be studied through epigenetic mechanisms such as DNA methylation. While various parental factors are known to increase risk for ASD, several studies have indicated that grandparental and greatgrandparental factors may also contribute. In animal studies, gestational exposure to certain environmental factors, such as insecticides, medications, and social stress, increases risk for altered behavioral phenotypes in multiple subsequent generations. Changes in DNA methylation, gene expression, and chromatin accessibility often accompany these altered behavioral phenotypes, with changes often appearing in genes that are important for neurodevelopment or have been previously implicated in ASD. One hypothesized mechanism for these phenotypic and methylation changes includes the transmission of DNA methylation marks at individual chromosomal loci from parent to offspring and beyond, called multigenerational epigenetic inheritance. Alternatively, intermediate metabolic phenotypes in the parental generation may confer risk from the original grandparental exposure to risk for ASD in grandchildren, mediated by DNA methylation. While hypothesized mechanisms require further research, the potential for multigenerational epigenetics assessments of ASD risk has implications for precision medicine as the field attempts to address the variable etiology and clinical signs of ASD by incorporating genetic, environmental, and lifestyle factors. In this review, we discuss the promise of multigenerational DNA methylation investigations in understanding the complex etiology of ASD.

\footnotetext{
Keywords: autism spectrum disorder, DNA methylation, multigenerational, transgenerational, epigenetics, metabolism, neurodevelopment, precision medicine
}

\section{INTRODUCTION}

Autism spectrum disorder (ASD) is a group of neurodevelopmental disorders that are characterized by deficits in social reciprocity and communication, restricted interests, and repetitive behavior. The prevalence of ASD has been steadily increasing over the past several decades, now affecting approximately one in 54 children in the United States (Maenner 2020). Most cases of ASD arise from a confluence of genetic predisposition, environmental factors, and gene-environment 
interactions (Bourgeron 2015), with the latter two potentially contributing to the apparent increase in prevalence in recent decades (Grether et al., 2009; King and Bearman 2009).

The Developmental Origins of Health and disease (DOHaD) hypothesis posits that environmental insults experienced during early development may impact the health of individuals later in life, as well as future generations. This framework fits the epidemiological and experimental findings that a wide range of environmental factors experienced in utero can affect risk for ASD. Prenatal risk factors with particularly strong evidence include advanced parental age, maternal medication use, and gestational diabetes (reviewed in Gardener et al., 2009; Karimi et al., 2017; Modabbernia et al., 2017), while folic acid may function as a protective factor (Levine et al., 2018). Due to the unequal exposure to risk factors and access to protective factors, this may generate an undue burden on underserved populations.

Further, the findings of several studies have indicated that certain environmental exposures may impact the health of individuals for multiple generations following the initial insult (reviewed in Breton et al., 2021). Human and animal studies are beginning to examine multigenerational risk factors for ASD, defined here as any factors that are non-genetic but may include demographic/social factors, chemical exposures, medications, and medical conditions in the generations prior to the individual diagnosed with ASD. The study of these potential multigenerational factors is warranted given the increase in ASD prevalence over the past several decades, which predominantly affects grandchildren of a population exposed to a surge of unregulated chemical pollutants in the mid 20th century (reviewed in Naidu et al., 2021).

Additionally, the field is working to identify potential mechanisms for transmission of risk through multiple generations. One of the most promising potential mechanisms is DNA methylation, an epigenetic mark that is hypothesized to be a mechanistic link between genetic and environmental risk (Law and Holland 2019), as well as a potential biomarker for ASD (Kimura et al., 2019; Zhu et al., 2019; Mordaunt et al., 2020; García-Ortiz et al., 2021; Garrido et al., 2021). Relatively few studies have examined DNA methylation in conjunction with grandparental or great-grandparental factors, despite the promising findings of existing multigenerational studies. This review describes the evidence for multigenerational risk for ASD in humans and animal models and explores the potential of DNA methylation for monitoring and/or counteracting multigenerational risk.

\subsection{Autism Spectrum Disorder}

ASD has no known single cause but numerous genetic and environmental factors that may individually contribute risk, and/or interact with one another. Estimates of heritability range widely, from $38 \%$ (Hallmayer et al., 2011) to $50 \%$ (Sandin et al., 2014), and upwards to 91\% (meta-analysis by Tick et al., 2016). Most inherited genetic risk is predicted to arise from common variants (Gaugler et al., 2014) that have small effect sizes but are predicted to act in combination and have been associated with other outcomes such as schizophrenia, depression, and educational attainment (Grove et al., 2019).
Rare de novo copy number and single nucleotide variants have been identified from exome sequencing of ASD and parent trios; these also overlap with variants associated with schizophrenia and intellectual disability (Iossifov et al., 2014). The genetic landscape of ASD has proven to be complex with hundreds of variants that may contribute to risk; however, to date no single gene or group of genes can predict or diagnose all cases of ASD.

Though ASD research has historically focused on genetic contributions to risk, newer studies have increasingly investigated environmental factors that may affect risk for ASD (reviewed in Bölte et al., 2019; Yoon et al., 2020). In line with the $\mathrm{DOHaD}$ hypothesis, studies have largely focused on prenatal and maternal factors, including demographics (e.g. parental age) (Reichenberg et al., 2006; King et al., 2009; Puleo et al., 2012); meta-analysis by Sandin et al. (2012), Taylor et al. (2019), maternal metabolic health (e.g. obesity and diabetes) (Li et al., 2016; Wang et al., 2019), chemical exposures (e.g. pesticides, air pollution, cigarette smoke) (Mitchell et al., 2012; meta-analysis by Jung et al., 2017; Granillo et al., 2019; meta-analysis by Chun et al., 2020), obstetric events (e.g. hypoxia) (Mann et al., 2010; Walker et al., 2015), and maternal medications (e.g. valproic acid) (Wiggs et al., 2020). Additionally, potential protective factors for ASD have been identified, including maternal folic acid (Levine et al., 2018) and dietary fat intake (Lyall et al., 2013). Prenatal and maternal factors have been thoroughly reviewed in previous publications (Gardener et al., 2009; Lyall et al., 2014; Wang et al., 2017; Hisle-Gorman et al., 2018; Katz et al., 2021).

\subsection{DNA Methylation}

The $\mathrm{DOHaD}$ hypothesis posits that the effects of early environmental exposures on health later in life may be mediated by epigenetic marks such as DNA methylation, which refers to the addition of a methyl group to carbon five on the pyrimidine ring of cytosine, forming 5-methylctyosine (5mC). In humans, DNA methylation occurs predominantly at CpG sites where the cytosine nucleotide on both strands is methylated, forming a symmetrical pattern. Approximately $70-80 \%$ of all cytosines in CpG nucleotides in the human genome are methylated (Kundaje et al., 2015), though this varies by cell type and location in the genome. Following development, global methylation levels remain nearly constant throughout the lifetime, though individual CpGs may change methylation status during further cellular differentiation (reviewed in Suelves et al., 2016), in response to extracellular signals (reviewed in Moore, Le, and Fan 2013), or in response to rhythmic cycles of diurnal metabolism (reviewed in Powell and LaSalle 2015). In contrast to gene expression which reflects the current state of cells, DNA methylation may reflect gene expression in other tissues (Gunasekara et al., 2019), in utero experiences, and past historical exposures of prior generations.

Multiple recent studies have identified a clear DNA methylation signature that can distinguish ASD from control DNA samples in multiple tissues, including sperm (Garrido et al., 2021), adult blood (Kimura et al., 2019), pediatric blood (GarcíaOrtiz et al., 2021), umbilical cord blood (Mordaunt et al., 2020), and placenta (Zhu et al., 2019). While these studies fall short of being able to accurately predict ASD from differential 


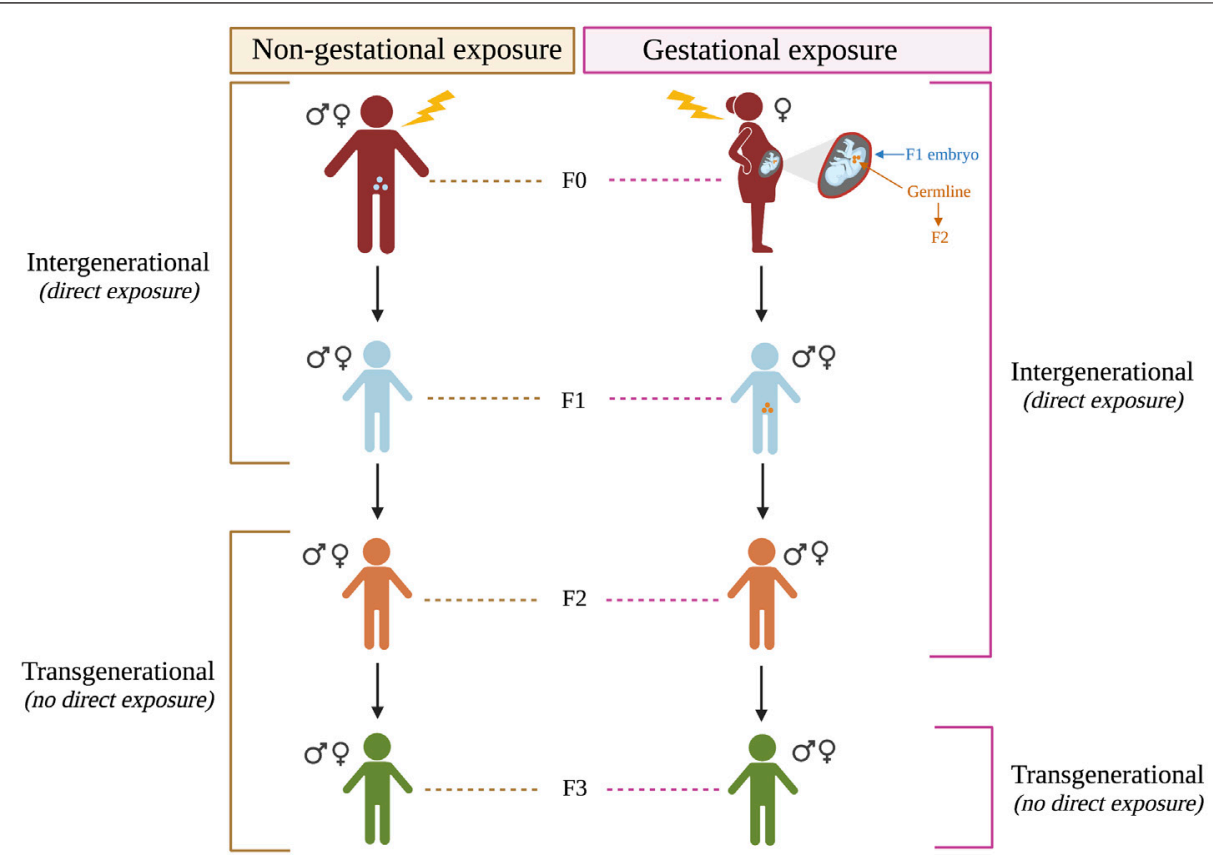

FIGURE 1 | Multigenerational effects following gestational and non-gestational exposures. Multigenerational includes intergenerational effects from direct exposure to an environmental result and transgenerational effects from indirect exposure (i.e. the generations beyond the directly-exposed generations).

methylation patterns, they do identify convergent genes such as MECP2 (Nagarajan et al., 2006; Liyanage et al., 2019; Lu et al., 2020) and GABRB3 (Wong et al., 2014). Research has yet to elucidate the mechanistic relationship between DNA methylation and ASD etiology or clinical variability but has shown a clear correlation between ASD diagnosis and altered patterns of DNA methylation across the genome.

\section{MULTIGENERATIONAL RISK FOR ASD}

In addition to the prenatal and maternal factors discussed above, recent human and animal studies have shown support for multigenerational risk for ASD. In this review, the term 'multigenerational' encompasses 'intergenerational' factors, which affect generations that are directly exposed to an environmental insult (whether as an adult, fetus, or gamete), and 'transgenerational' factors, which affect later generations who were not directly exposed (Figure 1) (reviewed in Heard and Martienssen 2014; Tuscher and Day 2019). If the directly exposed individual (F0) is not pregnant (non-gestational exposure), then the $\mathrm{F} 1$ generation has exposure from the gametes, so the F2 is the first generation not directly exposed. However, if the exposure occurs during pregnancy (gestational exposure) the first non-exposed generation is F3 since the fetus (F1) and their gametes (F2) are directly exposed to the insult. For a molecular mechanism to be transgenerational, it must produce effects in generations that were not directly exposed. While human studies have been primarily limited to intergenerational factors, several animal studies have examined potential transgenerational factors, as discussed in the next sections.

\subsection{Intergenerational Risk Factors}

Below is a discussion of potential risk factors for ASD that have been identified from three-generation human studies, with animal studies often supporting the findings. Behavioral changes in animals are used as a proxy for neurodevelopmental diagnoses in humans, though the measured phenotypes range across studies. Major categories of identified intergenerational risk factors are advanced age, medication use, chemical exposure, and social stress; both maternal and paternal lineages are implicated, though each shows distinct patterns.

\subsubsection{Age}

Advanced parental and grandparental age, particularly in the paternal line, has been one of the factors most strongly associated with ASD. For example, risk for ASD is increased if the individual's grandfather fathered children at an advanced age (Frans et al., 2013; Gao et al., 2020). This finding is independent of the parent's age at the grandchild's birth, suggesting that risk for ASD may develop over generations. In mice, advanced age of the F0 male during breeding led to increased ultrasound vocalization activity, decreased sociability, increased grooming activity, and increased anxiety-like responses in both the F1 and F2 generations (Sampino et al., 2014).

\subsubsection{Medication Use}

Certain medications, particularly when taken during pregnancy, may increase risk for ASD. For example, maternal grandmother use of diethylstylbestrol (DES), a synthetic form of estrogen, during pregnancy is associated with an increased risk of ADHD 
in male and female grandchildren (Kioumourtzoglou et al., 2018). A case-study of one family found that children and grandchildren from DES-free pregnancies did not develop psychiatric disorders, while those from DES-exposed pregnancies (from the same mother/grandmother) were highly likely to develop psychiatric disorders, including ASD (Soyer-Gobillard et al., 2021).

\subsubsection{Chemical Exposure}

Chemical exposures can range widely, from cigarette smoke to pesticides, and many may have effects on neurodevelopment (reviewed in Fujiwara et al., 2016). For instance, maternal grandmother smoking is associated with granddaughters having adverse scores in Social Communication and Repetitive Behavior measures, which are predictive phenotypes of ASD (Golding et al., 2017). Interestingly, this finding holds true only if the mother did not smoke during pregnancy. In mice, nicotine exposure induced attention-deficit/hyperactivity disorder (ADHD)-like behavior such as greater hyperactivity and risk-taking behavior in both the F1 and F2 generations (Buck et al., 2019). ADHD is a common co-occurrence of ASD (van der Meer et al., 2012; Russell et al., 2014) and the two diagnoses share overlapping phenotypes (Ronald et al., 2014).

Several insecticides have been identified as potential risk factors for ASD; permethrin, a widely used insecticide and known carcinogen, has been shown to impact the behavior of several generations following exposure. Exposure of F0 zebrafish to permethrin during early development through 28 days postfertilization affects F0 fertility and decreases activity of F1-F2 larvae (Blanc et al., 2020). Further, permethrin exposure is correlated with levels of lysophosphatidycholine, which is an important lipid for neurodevelopment.

\subsubsection{Social Stress}

Social-ecological factors, such as race, disability, insurance status, family financial burden, residence in a metropolitan area, and exposure to violence, have been associated with ASD prevalence in communities (Brisendine 2017). These studies are an important addition to experiments examining toxicological and biological factors, given the unequal burden of social stress on families and communities, and the continuation of many social factors through several generations. Social stressor experiments in rats can mimic those experienced by humans, such as family conflict and depressed maternal care. Chronic social stress administered during F0 lactation impaired the maternal care of both F0 and F1 dams. Further, both male and female F2 offspring from this model showed decreased social behavior, as well as increased juvenile oxytocin (females), decreased adult prolactin (females), and decreased corticosterone (males and females) (Babb et al., 2014).

\subsection{Transgenerational ASD Phenotypes and Gene Regulatory Changes}

Building upon intergenerational findings, many animal studies have examined transgenerational phenotypes, or those that extend beyond the directly exposed generations. Several such studies have also reported gene regulatory changes such as differential DNA methylation, gene expression, and chromatin accessibility. Given epigenetic marks' regulatory roles in gene expression (Jones 2012; Anastasiadi et al., 2018) and chromatin accessibility (Zhong et al., 2021), transcription level and chromatin changes may implicate DNA methylation as a potential mechanism conferring the effects of an environmental insult. While it is currently unclear whether changes in DNA methylation are causally related to multigenerational phenotypes, the studies discussed here show that increased investigation is worthwhile. Below are findings of studies that evaluate behavioral phenotypes as well as changes in DNA methylation, gene expression, and/or chromatin accessibility in at least three generations following a gestational exposure in an animal model.

\subsubsection{DNA Methylation}

Synthetic glucocorticoids are a medication administered to pregnant people at risk of delivering preterm, and have been shown to affect offspring neurocognitive and behavioral function and alter the fetal epigenome (Crudo et al., 2013). When F0 pregnant guinea pigs were exposed to glucocorticoids, response to stress and stress-associated behaviors were altered in the F1-F3 generations through both maternal and paternal lineages, though the effects diminished through generations (Moisiadis et al., 2017). These behavioral changes correlated with changes in hippocampal gene expression and DNA methylation, particularly in RNApol II binding regions of small non-coding RNA genes (Constantinof et al., 2019). Methylation changes that correlated with gene expression changes were enriched for enhancer regions, while methylation changes at individual CpGs were enriched for promoter regions of small non-coding RNAs (Constantinof et al., 2019). Interestingly, the F3 generation showed the highest number of differentially expressed genes and differentially methylated regions between exposed versus nonexposed guinea pigs, followed by the F1 generation and lastly by the F2 generation (Constantinof et al., 2019). This indicates that transcriptional changes in the hippocampus may increase across generations following glucocorticoid exposure.

\subsubsection{Gene Expression}

Valproic acid is a medication used to treat bipolar disorder and seizures and is a well-known risk factor for ASD in the offspring if taken while pregnant (Christensen et al., 2013; Wiggs et al., 2020). In mice studies of gestational valproic acid exposure, ASD-like phenotypes persisted through three generations following the exposure (Choi et al., 2016; Tartaglione et al., 2019). Transgenerational phenotypes included decreased sociability and increased marble burying (Choi et al., 2016), delayed righting reflex, increased motor activity, and reduced ultrasonic vocalizations (Tartaglione et al., 2019). Interestingly, the effects were stronger in the maternal lineage in Choi et al., but stronger in the paternal lineage in Tartaglione et al. Because different mouse strains were used in these studies (ICR versus $\mathrm{CD}-1$ ), these differences may suggest a gene by environment interaction. Accompanying the altered phenotypes, mice in the valproic acid-exposed lineages showed increased expression of genes previously implicated in ASD pathology, such as excitatory 
postsynaptic proteins PSD-95 and Pax6 (Choi et al., 2016). Increased expression of endogenous retroviruses was also observed (Tartaglione et al., 2019), potentially because throughout history endogenous retroviruses have inserted into the human genome following infection (reviewed in Grandi and Tramontano 2018), which is a known risk factor for ASD if experienced during pregnancy (Lee et al., 2015; Zerbo et al., 2015).

Maternal infection during pregnancy activates the immune response and generates inflammation, a process that may alter central nervous system development and give rise to neurodevelopmental disorders (reviewed in Han et al., 2021; Brown et al., 2014). These effects have been suggested to be mediated by epigenetic factors (reviewed in Bergdolt and Dunaevsky 2019), and may also have implications for ASDrelated behaviors past the F1 generation. Using a mouse model of prenatal immune activation with viral mimetic poly (I:C), Weber-Stadlbauer et al. showed reduced sociability and increased cued fear expression in three generations following exposure, via paternal lineage. Interestingly, the F2 and F3 offspring of immune-challenged ancestors showed increased behavioral despair, but this phenotype was not present in the F1 generation, again showing that novel phenotypes can appear multiple generations following exposure. Similarly, many genes were differentially expressed in the amygdalar complex of treatment versus control animals, with some changes being common across generations and some being generationspecific. 1,132 genes were differentially expressed in both F1 and F2 offspring, with enrichment for the DARPP-32 pathway, which has been previously associated with neuropsychiatric disorders (Kunii et al., 2014).

Bisphenol A (BPA) is an organic synthetic compound that exerts weak estrogenic activities and is associated with increased ASD risk (Stein et al., 2015; Hansen et al., 2021). ASD is diagnosed in males much more frequently than in females, at about a 4:1 ratio, and it has been posited that BPA exposure could play a role in ASD sex differences (Thongkorn et al., 2019). In mouse studies of gestational BPA exposure, delivered through chow, ASD-like phenotypes of reduced social recognition and activity persisted through three generations following the exposure (Wolstenholme et al., 2019). In F3 mice from the BPA lineage, expression levels of Shank1 were significantly different from controls in both the hypothalamus and lateral septum at embryonic stage, day-of-birth, and juvenile stage. Interestingly, the direction of change was not consistent across time points (Wolstenholme et al., 2019). Shank1 encodes a postsynaptic scaffolding protein, and mutations in this gene have been identified in individuals with ASD (reviewed in Monteiro and Feng 2017). In another study, differentially methylated regions were identified in the sperm of lineages of rats exposed to BPA, as well as DEET and TCDD (Manikkam et al., 2012), though behavioral phenotypes were not measured.

\subsubsection{Chromatin Accessibility}

Anesthetics have also been associated with neurobehavioral abnormalities following prenatal exposure (reviewed in Andropoulos 2018), particularly if the exposure occurs early in pregnancy (Cui et al., 2021). In a transgenerational mouse study modeling human anesthesia, gestational exposure to sevoflurane led to anxiety and impairments in social interaction in the following three generations through the paternal lineage (Wang et al., 2021). While 38\% of F1 showed these behavioral impairments, $44-47 \%$ of the F2 and F3 mice displayed them, again suggesting amplification across generations. To assess transcription factor distribution in the sperm genome of sevoflurane-treated versus control lines, the authors performed ATAC-sequencing, a method that analyzes chromatin accessibility using a Tn5 transposase that "tagments" open chromatin regions. ATAC-seq analysis of the F1 and F2 sperm showed 69 differentially accessible sites that are shared across the two generations of treated versus control lines. Differentially accessible sites in F1 sperm were enriched for genes involved in diseases of the nervous system and mental disorders and overlapped with ASD candidate genes such as Arid1b, Ntrk2, and Stmn2 (Wang et al., 2021). The authors suggested that changes in chromatin accessibility and transcription factor binding may prevent DNA re-methylation during reprogramming of the epiblast, leading to downstream changes in gene expression (Kremsky and Corces 2020; Wang et al., 2021). This mechanistic possibility is explored further in the next section.

\subsubsection{Summary of Transgenerational Findings}

Taken together, these studies show that DNA methylation, gene expression, and chromatin accessibility are often dysregulated in concert with altered behavioral phenotypes following a transgenerational exposure. Like the findings from intergenerational human studies, the maternal and paternal lineages in the transgenerational animal studies appeared to have different effects. Further, in several studies, the direction of change was not consistent across generations, and was often amplified in F3 compared to F1 and F2, indicating a potentially complex mechanism of transgenerational effects.

\section{POTENTIAL MECHANISMS FOR MULTIGENERATIONAL RISK FOR ASD}

While multigenerational changes in phenotype as well as DNA methylation, gene expression, and chromatin accessibility following an environmental insult have been well documented, the mechanisms to explain such results are less well established. Current evidence for multigenerational epigenetic inheritance is reviewed below, as well as literature pointing towards a potential alternative mechanism.

\subsection{Multigenerational Epigenetic Inheritance}

The most studied epigenetic mechanism for disease risk being transferred through generations is the maintenance of DNA methylation at some chromosomal loci that escape the normal erasure and reestablishment in the germline; this is termed multigenerational epigenetic inheritance (MEI) (or 
transgenerational epigenetic inheritance (TEI) if the mechanism extends to non-exposed generations). Though there is significant evidence for MEI in plants (Hauser et al., 2011), yeast and nematodes (Fabrizio et al., 2019) and zebrafish (Cavalieri and Spinelli 2017), there is not currently substantial evidence for MEI in mammals.

MEI is often thought to be unlikely because DNA methylation patterns are generally not maintained through meiotic divisions. During development, DNA methylation is erased and reestablished through two genome-wide demethylation events: 1) after fertilization of the zygote and 2) during the formation of primordial germ cells, which are direct precursors to gametes (reviewed in Zeng and Chen 2019). These erasures allow for totipotency while reestablishment of DNA methylation marks commits cells to a fate. However, DNA methylation of the mouse, pig, and human germlines has been shown to exhibit incomplete erasure, (Kearns et al., 2000; Sutherland et al., 2000; Tang et al., 2015; Guo et al., 2017; Gómez-Redondo et al., 2021), with primordial germ cells retaining approximately $10 \%$ of their methylation marks (Seisenberger et al., 2012) while the inner cell mass of the blastocyst retains approximately 20\% (L. Wang et al., 2014). This incomplete erasure indicates that some methylation marks are passed through the germline.

The most pronounced example of incomplete erasure occurs with imprinted genes, whereby DNA methylation marks prevent the expression of one parental allele, while the other parental allele is expressed. DNA methylation marks of imprinted genes are established in the germline of parents and retained through somatic cell divisions of the offspring. While some imprinted genes may have no biological relevance, others are necessary for proper development (reviewed in Tucci et al., 2019). Inappropriate imprinting may lead to neurodevelopmental disorders such as Angelman Syndrome and Prader-Willi Syndrome (reviewed in Butler 2020; Kalsner and Chamberlain 2015), which may also be caused by genetic defects such as uniparental disomy or chromosomal deletions.

Non-imprinted regions have also been shown to escape DNA methylation reprogramming (Tang et al., 2015). Mouse studies have shown that intracisternal A particles (IAPs) (Lane et al., 2003; Guibert et al., 2012), and LTR-ERV1 retroelements (Guibert et al., 2012) as well as other repetitive elements (Hajkova et al., 2002) escape reprogramming in primordial germ cells, while some retrotransposons escape reprogramming at the preimplantation embryo step (reviewed in (Zeng and Chen 2019). Interestingly, non-repetitive loci that escape reprogramming are enriched for genes involved in neurodevelopment and metabolism (Tang et al., 2015).

Regions may also experience MEI due to protection by bound transcription factors, as hypothesized by Kremsky and Corces 2020. One study found that $78 \%$ of CpGs maintain their methylation status following two rounds of reprogramming (Kremsky and Corces 2020). This faithful maintenance may be explained by transcription factors that bind $\mathrm{CpG}$ sites and prevent de-methylation and re-methylation, while unbound sites undergo reprogramming. Differential transcription factor binding may be explained by changes in chromatin accessibility (Wang et al., 2021). This possibility ties together the mechanisms of DNA methylation and chromatin accessibility to affect gene expression and potentially neurodevelopmental phenotypes.

In total, these studies raise the possibility of transmission of methylation marks at some loci through generations. This mechanism may help explain the ASD-related multigenerational phenotypes that follow environmental exposures, though further research is required.

\subsection{Alternative Mechanism for Multigenerational Effects: Intermediate Phenotypes in F1}

While MEI could be mechanistically involved with the transgenerational transmission of some phenotypes, alternative mechanisms are possible, particularly for intergenerational phenotypes. For example, an intermediate phenotype in the second generation (F1), such as metabolic dysfunction, could mediate the relationship between a gestational exposure in the first generation and adverse health effects, such as ASD, in the third generation or later (Figure 2).

Neurodevelopment and metabolism are closely linked, as the brain consumes $\sim 20 \%$ of the body's calories, while only representing $\sim 2 \%$ of body weight. Many patients diagnosed with ASD or related disorders such as ADHD suffer from metabolic abnormalities including obesity (Mota et al., 2020; reviewed in; Hill et al., 2015). Metabolism is adaptive to environmental changes, such as nutrient availability and chemical exposures (reviewed in Koyama et al., 2020). In humans, exposure to chemicals such as dichlorodiphenyltrichloroethane (DDT) (La Merrill et al., 2020) and polychlorinated biphenyls (PCBs) (S.-L. Wang et al., 2008; Philibert et al., 2009; Langer et al., 2014) increases risk for metabolic disorders such as obesity and diabetes, and DNA methylation patterns and dysregulated gene pathways are predicted to mediate this risk (Ghosh et al., 2014). In turn, metabolic dysfunction, including obesity, during pregnancy has been associated with increased risk of ASD in the offspring ( $\mathrm{Li}$ et al., 2016); this is again predicted to be mediated by altered DNA methylation (Lin et al., 2017). Additionally, regions that evaded genome-wide DNA demethylation in human primordial germ cells are enriched for genes that are expressed in the brain and are involved in neural development, as well as obesity-related phenotypes (Tang et al., 2015). These findings raise the possibility that metabolic dysfunction in the second generation could mediate environmental exposure in the first generation and increased risk for ASD in the third generation. This hypothesis, as well as other potential mechanisms, would benefit from studies specifically designed to evaluate their likelihood.

\section{GAPS IN THE RESEARCH AND FUTURE DIRECTIONS}

The studies discussed in this review demonstrate that ASD and ASD-related phenotypes may occur in multiple generations following an environmental insult. Further, these phenotypes 


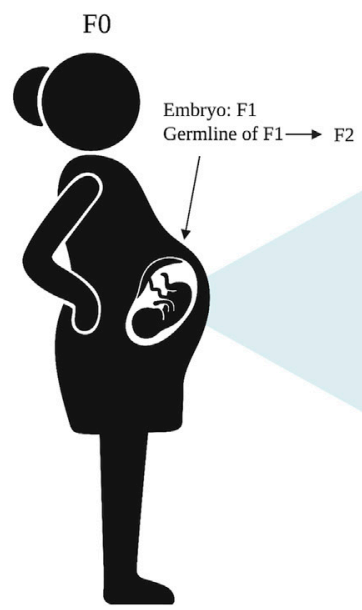

Gestational exposure

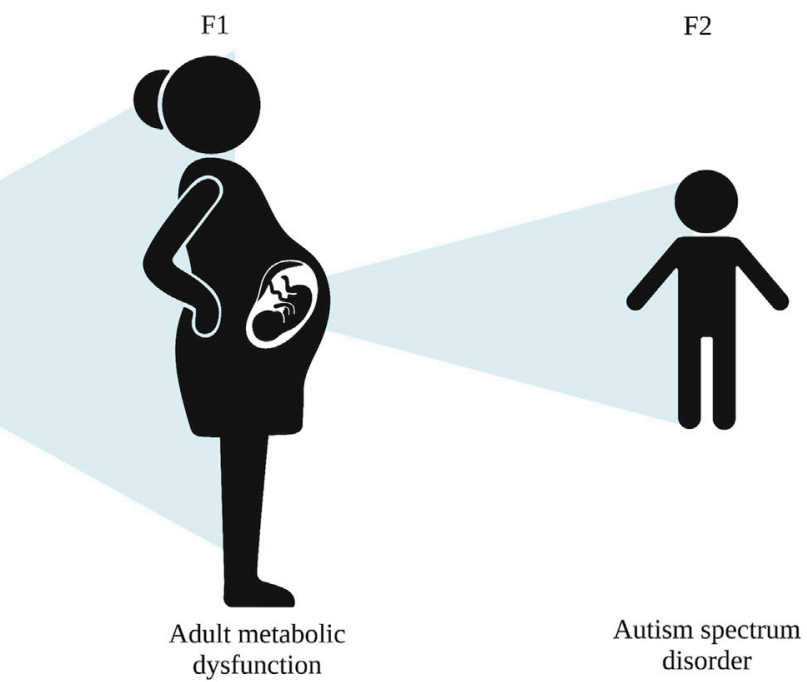

disorder

FIGURE 2 | An alternative mechanism for adverse health effects, such as ASD in the F2 generation, whereby an intermediate phenotype, such as metabolic dysfunction, in F1 mediated the F0 exposure and F2 phenotype.

may be reflected by altered DNA methylation, as well as gene expression and chromatin accessibility, even if these epigenetic changes are not causal. While the papers discussed in this review yield important findings, it is important to note the limitations of multigenerational studies. Human studies often lack sufficient controls and numbers of participants, as well as access to biospecimens and metadata from multiple generations and diverse populations. Animal studies address many of the deficiencies of multigenerational human studies but have yet to show causality of specific epigenetic changes with altered phenotypes. Additionally, the difficulty of translating findings from animal studies to humans remains a challenge, particularly when examining diseases of complex etiology and clinical phenotypes, such as ASD. To help fill these critical gaps we have recently developed a systems biology approach that integrates multiple variables of exposures, genetics, demographics, and social determinants of health with regions of co-methylation (Mordaunt et al., 2022).

Despite the challenges faced by multigenerational investigations, the findings discussed in this review have important implications for the field of precision medicine, which seeks to integrate genetic, environmental, and lifestyle data to customize healthcare. Precision medicine is particularly relevant to ASD given the current paucity of diagnostic and treatment tools, as well as the numerous genetic and environmental risk factors (reviewed in Loth et al., 2016). In recent years, methods have been developed to utilize epigenetic information in clinical settings, enhancing the effectiveness of precision medicine (BLUEPRINT consortium 2016). However, the field could be further advanced by incorporating multigenerational epigenetic information and phenotypes. For instance, identifying markers of in utero and historical exposures at birth could help identify infants at risk for ASD, enabling early interventions that have been associated with improved outcomes (reviewed in Masi et al., 2017). Epigenetic biomarkers may also help identify sub-types of ASD as well as potential treatment options (Masi et al., 2017).

There remain many gaps in the mechanistic understanding of multigenerational phenotypes and DNA methylation. Further studies and improved methods are needed to elucidate precise mechanisms of action of environmental factors, geneenvironment interactions, and multigenerational effects. The results of such studies may help to identify biomarkers and risk factors for disease, improving diagnostic and treatment practices as precision medicine develops.

\section{AUTHOR CONTRIBUTIONS}

JSM and JML conceived of, planned and wrote the manuscript. Both authors contributed to the article and approved the submitted version.

\section{FUNDING}

This publication was made possible by an NIEHS-funded predoctoral fellowship to JSM (T32 ES007059) and grant to JML (R01 ES029213). Its contents are solely the responsibility of the authors and do not necessarily represent the official views of the NIEHS or NIH.

\section{ACKNOWLEDGMENTS}

Figures 1, 2 created with BioRender.com. 


\section{REFERENCES}

Anastasiadi, D., Esteve-Codina, A., and Piferrer, F. (2018). Consistent Inverse Correlation between DNA Methylation of the First Intron and Gene Expression across Tissues and Species. Epigenetics \& Chromatin 11 (1), 37. doi:10.1186/ s13072-018-0205-1

Andropoulos, D. B. (2018). Effect of Anesthesia on the Developing Brain: Infant and Fetus. Fetal Diagn. Ther. 43 (1), 1-11. doi:10.1159/000475928

Babb, J. A., Carini, L. M., Spears, S. L., and Nephew, B. C. (2014). Transgenerational Effects of Social Stress on Social Behavior, Corticosterone, Oxytocin, and Prolactin in Rats. Horm. Behav. 65 (4), 386-393. doi:10.1016/j.yhbeh.2014. 03.005

Bergdolt, L., and Dunaevsky, A. (2019). Brain Changes in a Maternal Immune Activation Model of Neurodevelopmental Brain Disorders. Prog. Neurobiol. 175 (April), 1-19. doi:10.1016/j.pneurobio.2018.12.002

Blanc, M., Cormier, B., Hyötyläinen, T., Krauss, M., Scherbak, N., Cousin, X., et al. (2020). Multi- and Transgenerational Effects Following Early-Life Exposure of Zebrafish to Permethrin and Coumarin 47: Impact on Growth, Fertility, Behavior and Lipid Metabolism. Ecotoxicology Environ. Saf. 205 (December), 111348. doi:10.1016/j.ecoenv.2020.111348

BLUEPRINT consortium (2016). Quantitative Comparison of DNA Methylation Assays for Biomarker Development and Clinical Applications. Nat. Biotechnol. 34 (7), 726-737. doi:10.1038/nbt.3605

Bölte, S., Girdler, S., and Marschik, P. B. (2019). The Contribution of Environmental Exposure to the Etiology of Autism Spectrum Disorder. Cell. Mol. Life Sci. 76 (7), 1275-1297. doi:10.1007/s00018-018-2988-4

Bourgeron, T. (2015). From the Genetic Architecture to Synaptic Plasticity in Autism Spectrum Disorder. Nat. Rev. Neurosci. 16 (9), 551-563. doi:10.1038/ nrn3992

Breton, C. V., Landon, R., Kahn, L. G., Enlow, M. B., Peterson, A. K., Bastain, T., et al. (2021). Exploring the Evidence for Epigenetic Regulation of Environmental Influences on Child Health across Generations. Commun. Biol. 4 (June), 769. doi:10.1038/s42003-021-02316-6

Brisendine, Anne. Elizabeth. (2017). "Autism Spectrum Disorders and SocialEcological Models: Understanding How Context Drives Prevalence," (Birmingham: ProQuest Dissertations Publishing 10643156). Dissertation.

Brown, A. S., Sourander, A., Hinkka-Yli-Salomäki, S., McKeague, I. W., Sundvall, J., and Surcel, H.-M. (2014). Elevated Maternal C-Reactive Protein and Autism in a National Birth Cohort. Mol. Psychiatry 19 (2), 259-264. doi:10.1038/mp. 2012.197

Buck, J. M., Sanders, K. N., Wagema, C. R., Knopik, V. S., Stitzel, J. A., and O’Neill, H. C. (2019). Developmental Nicotine Exposure Precipitates Multigenerational Maternal Transmission of Nicotine Preference and ADHD-like Behavioral, Rhythmometric, Neuropharmacological, and Epigenetic Anomalies in Adolescent Mice. Neuropharmacology 149 (May), 66-82. doi:10.1016/j. neuropharm.2019.02.006

Butler, M. G. (2020). Imprinting Disorders in Humans: A Review. Curr. Opin. Pediatr. 32 (6), 719-729. doi:10.1097/MOP.0000000000000965

Cavalieri, V., and Spinelli, G. (2017). Environmental Epigenetics in Zebrafish. Epigenetics \& Chromatin 10 (1), 46. doi:10.1186/s13072-017-0154-0

Choi, C. S., Gonzales, E. L., Kim, K. C., Yang, S. M., Kim, J.-W., Mabunga, D. F., et al. (2016). The Transgenerational Inheritance of Autism-like Phenotypes in Mice Exposed to Valproic Acid during Pregnancy. Sci. Rep. 6 (1), 1-11. doi:10. 1038/srep36250

Christensen, J., Grønborg, T. K., Sørensen, M. J., Schendel, D., Parner, E. T., Pedersen, L. H., et al. (2013). Prenatal Valproate Exposure and Risk of Autism Spectrum Disorders and Childhood Autism. JAMA 309 (16), 1696-1703. doi:10.1001/jama.2013.2270

Chun, H., Leung, C., Wen, S. W., McDonald, J., and Shin, H. H. (2020). Maternal Exposure to Air Pollution and Risk of Autism in Children: A Systematic Review and Meta-Analysis. Environ. Pollut. 256 (January), 113307. doi:10.1016/j. envpol.2019.113307

Constantinof, A., Boureau, L., Moisiadis, V. G., Kostaki, A., Szyf, M., and Matthews, S. G. (2019). Prenatal Glucocorticoid Exposure Results in Changes in Gene Transcription and DNA Methylation in the Female Juvenile Guinea Pig Hippocampus Across Three Generations. Sci. Rep. 9 (December). doi:10.1038/s41598-019-54456-9
Crudo, A., Suderman, M., Moisiadis, V. G., Petropoulos, S., Kostaki, A., Hallett, M., et al. (2013). Glucocorticoid Programming of the Fetal Male Hippocampal Epigenome. Endocrinology 154 (3), 1168-1180. doi:10.1210/en.2012-1980

Cui, F.-h., Li, J., Li, K.-z., Xie, Y.-g., and Zhao, X.-l. (2021). Effects of Sevoflurane Exposure during Different Stages of Pregnancy on the Brain Development of Rat Offspring. J. Anesth. 35 (5), 654-662. doi:10.1007/s00540-021-02972-2

Fabrizio, P., Garvis, S., and Palladino, F. (2019). Histone Methylation and Memory of Environmental Stress. Cells 8 (4), 339. doi:10.3390/cells8040339

Frans, E. M., Sandin, S., Reichenberg, A., Långström, N., Lichtenstein, P., McGrath, J. J., et al. (2013). Autism Risk Across Generations. JAMA Psychiatry 70 (5), 516-521. doi:10.1001/jamapsychiatry.2013.1180

Fujiwara, T., Morisaki, N., Honda, Y., Sampei, M., and Tani, Y. (2016). Chemicals, Nutrition, and Autism Spectrum Disorder: A Mini-Review. Front. Neurosci. 10, 174. doi:10.3389/fnins.2016.00174

Gao, Y., Yu, Y., Xiao, J., Luo, J., Zhang, Y., Tian, Y., et al. (2020). Association of Grandparental and Parental Age at Childbirth with Autism Spectrum Disorder in Children. JAMA Netw. Open 3 (4), e202868. doi:10.1001/jamanetworkopen. 2020.2868

García-Ortiz, M. V., Torre-Aguilar, M. J. d. l., Morales-Ruiz, T., Gómez-Fernández, A., Flores-Rojas, K., Gil-Campos, M., et al. (2021). Analysis of Global and Local DNA Methylation Patterns in Blood Samples of Patients with Autism Spectrum Disorder. Front. Pediatr. 9, 1066. doi:10.3389/fped.2021.685310

Gardener, H., Spiegelman, D., and Buka, S. L. (2009). Prenatal Risk Factors for Autism: Comprehensive Meta-Analysis. Br. J. Psychiatry 195 (1), 7-14. doi:10. 1192/bjp.bp.108.051672

Garrido, N., Cruz, F., Egea, R. R., Simon, C., Sadler-Riggleman, I., Beck, D., et al. (2021). Sperm DNA Methylation Epimutation Biomarker for Paternal Offspring Autism Susceptibility. Clin. Epigenet 13 (January). doi:10.1186/ s13148-020-00995-2

Gaugler, T., Klei, L., Sanders, S. J., Bodea, C. A., Goldberg, A. P., Lee, A. B., et al. (2014). Most Genetic Risk for Autism Resides with Common Variation. Nat. Genet. 46 (8), 881-885. doi:10.1038/ng.3039

Ghosh, S., Murinova, L., Trnovec, T., Loffredo, C., Washington, K., Mitra, P., et al. (2014). Biomarkers Linking PCB Exposure and Obesity. Cpb 15 (11), 1058-1068. doi:10.2174/1389201015666141122203509

Golding, J., Ellis, G., Gregory, S., Birmingham, K., Iles-Caven, Y., Rai, D., et al. (2017). Grand-maternal Smoking in Pregnancy and Grandchild's Autistic Traits and Diagnosed Autism. Sci. Rep. 7 (April), 46179. doi:10.1038/srep46179

Gómez-Redondo, I., Planells, B., Cánovas, S., Ivanova, E., Kelsey, G., and Gutiérrez-Adán, A. (2021). Genome-Wide DNA Methylation Dynamics during Epigenetic Reprogramming in the Porcine Germline. Clin. Epigenet 13 (February), 27. doi:10.1186/s13148-021-01003-x

Grandi, N., and Tramontano, E. (2018). Human Endogenous Retroviruses Are Ancient Acquired Elements Still Shaping Innate Immune Responses. Front. Immunol. 9, 2039. doi:10.3389/fimmu.2018.02039

Granillo, L., Sethi, S., Keil, K. P., Lin, Y., Ozonoff, S., Iosif, A.-M., et al. (2019). Polychlorinated Biphenyls Influence on Autism Spectrum Disorder Risk in the MARBLES Cohort. Environ. Res. 171, 177-184. doi:10.1016/j.envres.2018. 12.061

Grether, J. K., Rosen, N. J., Smith, K. S., and Croen, L. A. (2009). Investigation of Shifts in Autism Reporting in the California Department of Developmental Services. J. Autism Dev. Disord. 39 (10), 1412-1419. doi:10.1007/s10803-0090754-z

Grove, J., Ripke, S., Als, T. D., Mattheisen, M., Walters, R. K., Won, H., et al. (2019). Identification of Common Genetic Risk Variants for Autism Spectrum Disorder. Nat. Genet. 51 (3), 431-444. doi:10.1038/s41588-019-0344-8

Guibert, S., Forné, T., and Weber, M. (2012). Global Profiling of DNA Methylation Erasure in Mouse Primordial Germ Cells. Genome Res. 22 (4), 633-641. doi:10 1101/gr.130997.111

Gunasekara, C. J., Scott, C. A., Laritsky, E., Baker, M. S., MacKay, H., Duryea, J. D., et al. (2019). A Genomic Atlas of Systemic Interindividual Epigenetic Variation in Humans. Genome Biol. 20 (June). doi:10.1186/s13059-019-1708-1

Guo, H., Hu, B., Yan, L., Yong, J., Wu, Y., Gao, Y., et al. (2017). DNA Methylation and Chromatin Accessibility Profiling of Mouse and Human Fetal Germ Cells. Cell Res 27 (2), 165-183. doi:10.1038/cr.2016.128

Hajkova, P., Erhardt, S., Lane, N., Haaf, T., El-Maarri, O., Reik, W., et al. (2002). Epigenetic Reprogramming in Mouse Primordial Germ Cells. Mech. Dev. 117 (1-2), 15-23. doi:10.1016/s0925-4773(02)00181-8 
Hallmayer, J., Cleveland, S., Torres, A., Phillips, J., Cohen, B., Torigoe, T., et al. (2011). Genetic Heritability and Shared Environmental Factors Among Twin Pairs with Autism. Arch. Gen. Psychiatry 68 (11), 1095-1102. doi:10.1001/ archgenpsychiatry.2011.76

Han, V. X., Patel, S., Jones, H. F., and Dale, R. C. (2021). Maternal Immune Activation and Neuroinflammation in Human Neurodevelopmental Disorders. Nat. Rev. Neurol. 17 (9), 564-579. doi:10.1038/s41582-021-00530-8

Hansen, J. B., Bilenberg, N., Timmermann, C. A. G., Jensen, R. C., Frederiksen, H., Andersson, A.-M., et al. (2021). Prenatal Exposure to Bisphenol A and Autisticand ADHD-Related Symptoms in Children Aged 2 And5 Years from the Odense Child Cohort. Environ. Health 20 (1), 24. doi:10.1186/s12940-021-00709-y

Hauser, M.-T., Aufsatz, W., Jonak, C., and Luschnig, C. (2011). Transgenerational Epigenetic Inheritance in Plants. Biochim. Biophys. Acta (Bba) - Gene Regul. Mech. 1809 (8), 459-468. doi:10.1016/j.bbagrm.2011.03.007

Heard, E., and Martienssen, R. A. (2014). Transgenerational Epigenetic Inheritance: Myths and Mechanisms. Cell 157 (1), 95-109. doi:10.1016/j.cell. 2014.02.045

Hill, A. P., Zuckerman, K. E., and Fombonne, E. (2015). Obesity and Autism. Pediatrics 136 (6), 1051-1061. doi:10.1542/peds.2015-1437

Hisle-Gorman, E., Susi, A., Stokes, T., Gorman, G., Erdie-Lalena, C., and Nylund, C. M. (2018). Prenatal, Perinatal, and Neonatal Risk Factors of Autism Spectrum Disorder. Pediatr. Res. 84 (2), 190-198. doi:10.1038/pr.2018.23

Iossifov, I., O’Roak, B. J., SandersMichael Ronemus, S. J., Ronemus, M., Krumm, N., Levy, D., et al. (2014). The Contribution of De Novo Coding Mutations to Autism Spectrum Disorder. Nature 515 (7526), 216-221. doi:10.1038/ nature13908

Jones, P. A. (2012). Functions of DNA Methylation: Islands, Start Sites, Gene Bodies and beyond. Nat. Rev. Genet. 13 (7), 484-492. doi:10.1038/nrg3230

Jung, Y., Lee, A. M., McKee, S. A., and Picciotto, M. R. (2017). Maternal Smoking and Autism Spectrum Disorder: Meta-Analysis with Population Smoking Metrics as Moderators. Sci. Rep. 7 (1), 4315. doi:10.1038/s41598-017-04413-1

Kalsner, L., and Chamberlain, S. J. (2015). Prader-Willi, Angelman, and 15q11Q13 Duplication Syndromes. Pediatr. Clin. North America 62 (3), 587-606. doi:10.1016/j.pcl.2015.03.004

Karahmadi, M., Karimi, P., Kamali, E., and Mousavi, S. (2017). Environmental Factors Influencing the Risk of Autism. J. Res. Med. Sci. 22 (February), 27. doi:10.4103/1735-1995.200272

Katz, J., Reichenberg, A., and Kolevzon, A. (2021). Prenatal and Perinatal Metabolic Risk Factors for Autism: A Review and Integration of Findings from Population-Based Studies. Curr. Opin. Psychiatry 34 (2), 94-104. doi:10. 1097/YCO.0000000000000673

Kearns, M., Preis, J., McDonald, M., Morris, C., and Whitelaw, E.(2000). Complex Patterns of Inheritance of an Imprinted Murine Transgene Suggest Incomplete Germline Erasure. Nucleic Acids Res. 28 (17), 3301-3309. doi:10.1093/nar/28. 17.3301

Kimura, R., Nakata, M., Funabiki, Y., Suzuki, S., Awaya, T., Murai, T., et al. (2019). An Epigenetic Biomarker for Adult High-Functioning Autism Spectrum Disorder. Sci. Rep. 9 (1), 13662. doi:10.1038/s41598-019-50250-9

King, M., and Bearman, P. (2009). Diagnostic Change and the Increased Prevalence of Autism. Int. J. Epidemiol. 38 (5), 1224-1234. doi:10.1093/ije/dyp261

King, M. D., Fountain, C., Dakhlallah, D., and Bearman, P. S. (2009). Estimated Autism Risk and Older Reproductive Age. Am. J. Public Health 99 (9), 1673-1679. doi:10.2105/AJPH.2008.149021

Kioumourtzoglou, M.-A., Coull, B. A., O’Reilly, É. J., Ascherio, A., and Weisskopf, M. G. (2018). Association of Exposure to Diethylstilbestrol during Pregnancy with Multigenerational Neurodevelopmental Deficits. JAMA Pediatr. 172 (7), 670-677. doi:10.1001/jamapediatrics.2018.0727

Koyama, T., Texada, M. J., Halberg, K. A., and Rewitz, K. (2020). Metabolism and Growth Adaptation to Environmental Conditions in Drosophila. Cel. Mol. Life Sci. 77 (22), 4523-4551. doi:10.1007/s00018-020-03547-2

Kremsky, I., and Corces, V. G. (2020). Protection from DNA Re-methylation by Transcription Factors in Primordial Germ Cells and Pre-implantation Embryos Can Explain Trans-generational Epigenetic Inheritance. Genome Biol. 21 (1), 118. doi:10.1186/s13059-020-02036-w

Kundaje, A., Meuleman, W., Ernst, J., Bilenky, M., Yen, A., Heravi-Moussavi, A., et al. (2015). Integrative Analysis of 111 Reference Human Epigenomes. Nature 518 (7539), 317-330. doi:10.1038/nature14248
Kunii, Y., Hyde, T. M., Ye, T., Li, C., Kolachana, B., Dickinson, D., et al. (2014). Revisiting DARPP-32 in Postmortem Human Brain: Changes in Schizophrenia and Bipolar Disorder and Genetic Associations with T-DARPP-32 Expression. Mol. Psychiatry 19 (2), 192-199. doi:10.1038/mp.2012.174

La Merrill, M. A., Krigbaum, N. Y., Cirillo, P. M., and Cohn, B. A., (2020). Association between Maternal Exposure to the Pesticide Dichlorodiphenyltrichloroethane (DDT) and Risk of Obesity in Middle Age. Int. J. Obes. 44, 1723-1732. doi:10.1038/s41366-020-0586-7

Lane, N., Dean, W., Erhardt, S., Hajkova, P., Surani, A., Walter, J. r., et al. (2003). Resistance of IAPs to Methylation Reprogramming May Provide a Mechanism for Epigenetic Inheritance in the Mouse. genesis 35 (2), 88-93. doi:10.1002/ gene. 10168

Langer, P., Ukropec, J., Kocan, A., Drobna, B., Radikova, Z., Huckova, M., et al. (2014). Obesogenic and Diabetogenic Impact of High Organochlorine Levels (HCB, P,p'-DDE, PCBs) on Inhabitants in the Highly Polluted Eastern Slovakia. endo 48 (1), 17-24. doi:10.4149/endo_2014_01_17

Law, P.-P., and Holland, M. L. (2019). DNA Methylation at the Crossroads of Gene and Environment Interactions. Essays Biochem. 63 (6), 717-726. doi:10.1042/ EBC20190031

Lee, B. K., Magnusson, C., Gardner, R. M., Blomström, A., Newschaffer, C. J., Burstyn, I., et al. (2015). Maternal Hospitalization with Infection during Pregnancy and Risk of Autism Spectrum Disorders. Brain Behav. Immun. 44 (February), 100-105. doi:10.1016/j.bbi.2014.09.001

Levine, S. Z., Kodesh, A., Viktorin, A., Smith, L., Uher, R., Reichenberg, A., et al. (2018). Association of Maternal Use of Folic Acid and Multivitamin Supplements in the Periods before and during Pregnancy with the Risk of Autism Spectrum Disorder in Offspring. JAMA Psychiatry 75 (2), 176-184. doi:10.1001/jamapsychiatry.2017.4050

Li, M., Fallin, M. D., Riley, A., Landa, R., Walker, S. O., Silverstein, M., et al. (2016). The Association of Maternal Obesity and Diabetes with Autism and Other Developmental Disabilities. Pediatrics 137 (2). doi:10.1542/peds.20152206

Lin, X., Lim, I. Y., Wu, Y., Teh, A. L., Chen, L., Aris, I. M., et al. (2017). Developmental Pathways to Adiposity Begin before Birth and Are Influenced by Genotype, Prenatal Environment and Epigenome. BMC Med. 15 (March). doi:10.1186/s12916-017-0800-1

Liyanage, V. R. B., Olson, C. O., Zachariah, R. M., Davie, J. R., and Rastegar, M. (2019). DNA Methylation Contributes to the Differential Expression Levels of Mecp2 in Male Mice Neurons and Astrocytes. Ijms 20 (8), 1845. doi:10.3390/ ijms 20081845

Loth, E., Murphy, D. G., and Spooren, W. (2016). Defining Precision Medicine Approaches to Autism Spectrum Disorders: Concepts and Challenges. Front. Psychiatry 7 (November), 188. doi:10.3389/fpsyt. 2016.00188

Lu, Z., Liu, Z., Mao, W., Wang, X., Zheng, X., Chen, S., et al. (2020). LocusSpecific DNA Methylation of Mecp2 Promoter Leads to Autism-like Phenotypes in Mice. Cell Death Dis 11 (2), 1-11. doi:10.1038/s41419$020-2290-x$

Lyall, K., Munger, K. L., O’Reilly, É. J., Santangelo, S. L., and Ascherio, A. (2013). Maternal Dietary Fat Intake in Association with Autism Spectrum Disorders. Am. J. Epidemiol. 178 (2), 209-220. doi:10.1093/aje/kws433

Lyall, K., Schmidt, R. J., and Hertz-Picciotto, I. (2014). Maternal Lifestyle and Environmental Risk Factors for Autism Spectrum Disorders. Int. J. Epidemiol. 43 (2), 443-464. doi:10.1093/ije/dyt282

Maenner, M. J., Shaw, K. A., Baio, J., Washington, A., Patrick, M., DiRienzo, M., et al. (2020). Prevalence of Autism Spectrum Disorder Among Children Aged 8 Years - Autism and Developmental Disabilities Monitoring Network, 11 Sites, United States, 2016. MMWR Surveill. Summ. 69, 1-12. doi:10.15585/mmwr. ss6904al

Manikkam, M., Guerrero-Bosagna, C., Tracey, R., Haque, M. M., and Skinner, M. K. (2012). Transgenerational Actions of Environmental Compounds on Reproductive Disease and Identification of Epigenetic Biomarkers of Ancestral Exposures. PLoS One 7 (2), e31901. doi:10.1371/journal.pone. 0031901

Mann, J. R., McDermott, S., Bao, H., Hardin, J., and Gregg, A. (2010). PreEclampsia, Birth Weight, and Autism Spectrum Disorders. J. Autism Dev. Disord. 40 (5), 548-554. doi:10.1007/s10803-009-0903-4 
Masi, A., DeMayo, M. M., Glozier, N., and Guastella, A. J. (2017). An Overview of Autism Spectrum Disorder, Heterogeneity and Treatment Options. Neurosci. Bull. 33 (2), 183-193. doi:10.1007/s12264-017-0100-y

Mitchell, M. M., Woods, R., Chi, L.-H., Schmidt, R. J., Pessah, I. N., Kostyniak, P. J., et al. (2012). Levels of Select PCB and PBDE Congeners in Human Postmortem Brain Reveal Possible Environmental Involvement in 15q11-Q13 Duplication Autism Spectrum Disorder. Environ. Mol. Mutagen. 53 (8), 589-598. doi:10. $1002 / \mathrm{em} .21722$

Modabbernia, A., Velthorst, E., and Reichenberg, A. (2017). Environmental Risk Factors for Autism: An Evidence-Based Review of Systematic Reviews and Meta-Analyses. Mol. Autism 8 (1), 13. doi:10.1186/s13229-017-0121-4

Moisiadis, V. G., Constantinof, A., Kostaki, A., Szyf, M., and Matthews, S. G. (2017). Prenatal Glucocorticoid Exposure Modifies Endocrine Function and Behaviour for 3 Generations Following Maternal and Paternal Transmission. Sci. Rep. 7 (September), 11814. doi:10.1038/s41598-017-11635-w

Monteiro, P., and Feng, G. (2017). SHANK Proteins: Roles at the Synapse and in Autism Spectrum Disorder. Nat. Rev. Neurosci. 18 (3), 147-157. doi:10.1038/ nrn.2016.183

Moore, L. D., Le, T., and Fan, G. (2013). DNA Methylation and its Basic Function. Neuropsychopharmacol 38 (1), 23-38. doi:10.1038/npp.2012.112

Mordaunt, C. E., Jianu, J. M., Laufer, B. I., Zhu, Y., Hwang, H., Dunaway, K. W., et al. (2020). Cord Blood DNA Methylome in Newborns Later Diagnosed with Autism Spectrum Disorder Reflects Early Dysregulation of Neurodevelopmental and X-Linked Genes. Genome Med. 12 (October). doi:10.1186/s13073-020-00785-8

Mordaunt, C. E., Mouat, J. S., Schmidt, R. J., and LaSalle, J. M. (2022). Comethyl: A Network-Based Methylome Approach to Investigate the Multivariate Nature of Health and Disease. Brief. Bioinform, bbab554. doi:10.1093/bib/bbab554

Mota, N. R., Poelmans, G., Klein, M., Torrico, B., Fernàndez-Castillo, N., Cormand, B., et al. (2020). Cross-Disorder Genetic Analyses Implicate Dopaminergic Signaling as a Biological Link between Attention-Deficit/Hyperactivity Disorder and Obesity Measures. Neuropsychopharmacol. 45 (7), 1188-1195. doi:10.1038/s41386-019-0592-4

Nagarajan, R. P., Hogart, A. R., Gwye, Y., Martin, M. R., and LaSalle, J. M. (2006). Reduced MeCP2 Expression Is Frequent in Autism Frontal Cortex and Correlates with Aberrant MECP2 Promoter Methylation. Epigenetics 1 (4), e1-11. doi:10.4161/epi.1.4.3514

Naidu, R., Biswas, B., Willett, I. R., Cribb, J., Singh, B. K., Nathanail, C. P., et al. (2021). Chemical Pollution: A Growing Peril and Potential Catastrophic Risk to Humanity. Environ. Int. 156 (November), 106616. doi:10.1016/j.envint.2021. 106616

Philibert, A., Schwartz, H., and Mergler, D. (2009). An Exploratory Study of Diabetes in a First Nation Community with Respect to Serum Concentrations of P,p'-DDE and PCBs and Fish Consumption. Ijerph 6 (12), 3179-3189. doi:10. 3390/ijerph6123179

Powell, W. T., and LaSalle, J. M. (2015). Epigenetic Mechanisms in Diurnal Cycles of Metabolism and Neurodevelopment. Hum. Mol. Genet. 24 (R1), R1-R9. doi:10.1093/hmg/ddv234

Puleo, C. M., Schmeidler, J., Reichenberg, A., Kolevzon, A., Soorya, L. V., Buxbaum, J. D., et al. (2012). Advancing Paternal Age and Simplex Autism. Autism 16 (4), 367-380. doi:10.1177/1362361311427154

Reichenberg, A., Gross, R., Weiser, M., Bresnahan, M., Silverman, J., Harlap, S., et al. (2006). Advancing Paternal Age and Autism. Arch. Gen. Psychiatry 63 (9), 1026-1032. doi:10.1001/archpsyc.63.9.1026

Ronald, A., Larsson, H., Anckarsäter, H., and Lichtenstein, P. (2014). Symptoms of Autism and ADHD: A Swedish Twin Study Examining Their Overlap. J. Abnormal Psychol. 123 (2), 440-451. doi:10.1037/a0036088

Russell, G., Rodgers, L. R., Ukoumunne, O. C., and Ford., T. (2014). Prevalence of ParentReported ASD and ADHD in the UK: Findings from the Millennium Cohort Study. J. Autism Dev. Disord. 44 (1), 31-40. doi:10.1007/s10803-013-1849-0

Sampino, S., Juszczak, G. R., Zacchini, F., Swiergiel, A. H., Modlinski, J. A., Loi, P., et al. (2014). Grand-Paternal Age and the Development of Autism-like Symptoms in Mice Progeny. Transl Psychiatry 4 (4), e386. doi:10.1038/tp. 2014.27

Sandin, S., Hultman, C. M., Kolevzon, A., Gross, R., MacCabe, J. H., and Reichenberg, A. (2012). Advancing Maternal Age Is Associated with Increasing Risk for Autism: A Review and Meta-Analysis. J. Am. Acad. Child Adolesc. Psychiatry 51 (5), 477-486. e1. doi:10.1016/j.jaac.2012.02.018
Sandin, S., Lichtenstein, P., Kuja-Halkola, R., Larsson, H., Hultman, C. M., and Reichenberg, A. (2014). The Familial Risk of Autism. JAMA 311 (17), 1770-1777. doi:10.1001/jama.2014.4144

Seisenberger, S., Andrews, S., Krueger, F., Arand, J., Walter, J., Santos, F., et al. (2012). The Dynamics of Genome-wide DNA Methylation Reprogramming in Mouse Primordial Germ Cells. Mol. Cel 48 (6), 849-862. doi:10.1016/j.molcel. 2012.11.001

Soyer-Gobillard, M.-O., Gaspari, L., Paris, F., Kalfa, N., Hamamah, S., Courtet, P., et al. (2021). Prenatal Exposure to Diethylstilbestrol and Multigenerational Psychiatric Disorders: An Informative Family. Ijerph 18 (19), 9965. doi:10.3390/ ijerph18199965

Stein, T. P., Schluter, M. D., Steer, R. A., Guo, L., and Ming, X. (2015). Bisphenol A Exposure in Children with Autism Spectrum Disorders. Autism Res. 8 (3), 272-283. doi:10.1002/aur.1444

Suelves, M., Carrió, E., Núñez-Álvarez, Y., and Peinado, M. A. (2016). DNA Methylation Dynamics in Cellular Commitment and Differentiation. Brief. Funct. Genomics 15 (6), elw017-53. doi:10.1093/bfgp/elw017

Sutherland, H. G. E., Kearns, M., Morgan, H. D., Headley, A. P., Morris, C., Martin, D. I. K., et al. (2000). Reactivation of Heritably Silenced Gene Expression in Mice. Mamm. Genome 11 (5), 347-355. doi:10.1007/s003350010066

Tang, W. W. C., Dietmann, S., Irie, N., Leitch, H. G., Floros, V. I., Bradshaw, C. R., et al. (2015). A Unique Gene Regulatory Network Resets the Human Germline Epigenome for Development. Cell 161 (6), 1453-1467. doi:10.1016/j.cell.2015. 04.053

Tartaglione, A. M., Cipriani, C., Chiarotti, F., Perrone, B., Balestrieri, E., Matteucci, C., et al. (2019). Early Behavioral Alterations and Increased Expression of Endogenous Retroviruses Are Inherited Across Generations in Mice Prenatally Exposed to Valproic Acid. Mol. Neurobiol. 56 (5), 3736-3750. doi:10.1007/ s12035-018-1328-x

Taylor, J. L., Debost, J.-C. P. G., Morton, S. U., Wigdor, E. M., Heyne, H. O., Lal, D., et al. (2019). Paternal-Age-Related De Novo Mutations and Risk for Five Disorders. Nat. Commun. 10 (1), 3043. doi:10.1038/s41467-019-11039-6

Thongkorn, S., Kanlayaprasit, S., Jindatip, D., Tencomnao, T., Hu, V. W., and Sarachana, T. (2019). Sex Differences in the Effects of Prenatal Bisphenol A Exposure on Genes Associated with Autism Spectrum Disorder in the Hippocampus. Sci. Rep. 9 (1), 3038. doi:10.1038/s41598-019-39386-w

Tick, B., Bolton, P., Happé, F., Rutter, M., and Rijsdijk, F. (2016). Heritability of Autism Spectrum Disorders: A Meta-Analysis of Twin Studies. J. Child. Psychol. Psychiatr. 57 (5), 585-595. doi:10.1111/jcpp.12499

Tucci, V., Isles, A. R., Kelsey, G., Ferguson-Smith, A. C., Tucci, V., Bartolomei, M. S., et al. (2019). Genomic Imprinting and Physiological Processes in Mammals. Cell 176 (5), 952-965. doi:10.1016/j.cell.2019.01.043

Tuscher, J. J., and Day, J. J. (2019). Multigenerational Epigenetic Inheritance: One Step Forward, Two Generations Back. Neurobiol. Dis. 132 (December), 104591. doi:10.1016/j.nbd.2019.104591

van der Meer, J. M. J., Oerlemans, A. M., van Steijn, D. J., Lappenschaar, M. G. A., de Sonneville, L. M. J., Buitelaar, J. K., et al. (2012). Are Autism Spectrum Disorder and Attention-Deficit/Hyperactivity Disorder Different Manifestations of One Overarching Disorder? Cognitive and Symptom Evidence from a Clinical and Population-Based Sample. J. Am. Acad. Child Adolesc. Psychiatry 51 (11), 1160-1172. e3. doi:10.1016/j.jaac. 2012.08.024

Walker, C. K., Krakowiak, P., Baker, A., Hansen, R. L., Ozonoff, S., and HertzPicciotto, I. (2015). Preeclampsia, Placental Insufficiency, and Autism Spectrum Disorder or Developmental Delay. JAMA Pediatr. 169 (2), 154-162. doi:10. 1001/jamapediatrics.2014.2645

Wang, C., Geng, H., Liu, W., and Zhang, G. (2017). Prenatal, Perinatal, and Postnatal Factors Associated with Autism. Medicine 96 (18), e6696. doi:10. 1097/MD.0000000000006696

Wang, H.-L. V., Forestier, S., and Corces, V. G. (2021). Exposure to Sevoflurane Results in Changes of Transcription Factor Occupancy in Sperm and Inheritance of Autism†. Biol. Reprod. 105, 705-719. doi:10.1093/biolre/ioab097ioab097 (May)

Wang, L., Zhang, J., Duan, J., Gao, X., Zhu, W., Lu, X., et al. (2014). Programming and Inheritance of Parental DNA Methylomes in Mammals. Cell 157 (4), 979-991. doi:10.1016/j.cell.2014.04.017

Wang, S.-L., Tsai, P.-C., Yang, C.-Y., and Leon Guo, Y. (2008). Increased Risk of Diabetes and Polychlorinated Biphenyls and Dioxins. Diabetes Care 31 (8), 1574-1579. doi:10.2337/dc07-2449 
Wang, X., Lu, J., Xie, W., Lu, X., Liang, Y., Li, M., et al. (2019). Maternal Diabetes Induces Autism-like Behavior by Hyperglycemia-Mediated Persistent Oxidative Stress and Suppression of Superoxide Dismutase 2. Proc. Natl. Acad. Sci. USA 116 (47), 23743-23752. doi:10.1073/pnas.1912625116

Wiggs, K. K., Rickert, M. E., Sujan, A. C., Quinn, P. D., Larsson, H., Lichtenstein, P., et al. (2020). Antiseizure Medication Use during Pregnancy and Risk of ASD and ADHD in Children. Neurology 95 (24), e3232-e3240. doi:10.1212/WNL. 0000000000010993

Wolstenholme, J. T., Drobná, Z., Henriksen, A. D., Goldsby, J. A., Stevenson, R., Irvin, J. W., et al. (2019). Transgenerational Bisphenol A Causes Deficits in Social Recognition and Alters Postsynaptic Density Genes in Mice. Endocrinology 160 (8), 1854-1867. doi:10.1210/en.2019-00196

Wong, C. C. Y., Meaburn, E. L., Ronald, A., Price, T. S., Jeffries, A. R., Schalkwyk, L. C., et al. (2014). Methylomic Analysis of Monozygotic Twins Discordant for Autism Spectrum Disorder and Related Behavioural Traits. Mol. Psychiatry 19 (4), 495-503. doi:10.1038/mp.2013.41

Yoon, S. H., Choi, J., Lee, W. J., and Do, J. T. (2020). Genetic and Epigenetic Etiology Underlying Autism Spectrum Disorder. Jcm 9 (4), 966. doi:10.3390/ jcm9040966

Zeng, Y., and Chen, T. (2019). DNA Methylation Reprogramming during Mammalian Development. Genes 10 (4), 257. doi:10.3390/genes 10040257

Zerbo, O., Qian, Y., Yoshida, C., Grether, J. K., Van de Water, J., and Croen, L. A. (2015). Maternal Infection during Pregnancy and Autism Spectrum Disorders. J. Autism Dev. Disord. 45 (12), 4015-4025. doi:10.1007/s10803013-2016-3
Zhong, Z., Feng, S., Duttke, S. H., Potok, M. E., Zhang, Y., Gallego-Bartolomé, J., et al. (2021). DNA Methylation-Linked Chromatin Accessibility Affects Genomic Architecture in Arabidopsis. Proc. Natl. Acad. Sci. USA 118 (5), e2023347118. doi:10.1073/pnas.2023347118

Zhu, Y., Mordaunt, C. E., Yasui, D. H., Marathe, R., Coulson, R. L., Dunaway, K. W., et al. (2019). Placental DNA Methylation Levels at CYP2E1 and IRS2 Are Associated with Child Outcome in a Prospective Autism Study. Hum. Mol. Genet. 28 (16), 2659-2674. doi:10.1093/hmg/ddz084

Conflict of Interest: The authors declare that the research was conducted in the absence of any commercial or financial relationships that could be construed as a potential conflict of interest.

Publisher's Note: All claims expressed in this article are solely those of the authors and do not necessarily represent those of their affiliated organizations, or those of the publisher, the editors and the reviewers. Any product that may be evaluated in this article, or claim that may be made by its manufacturer, is not guaranteed or endorsed by the publisher.

Copyright (c) 2022 Mouat and LaSalle. This is an open-access article distributed under the terms of the Creative Commons Attribution License (CC BY). The use, distribution or reproduction in other forums is permitted, provided the original author(s) and the copyright owner(s) are credited and that the original publication in this journal is cited, in accordance with accepted academic practice. No use, distribution or reproduction is permitted which does not comply with these terms. 